\title{
Erinnerungen an Hermann Sahli (1856-1933)
}

Wir feiern den 100. Geburtstag von Hermann Sahli, geboren am 23. Mai 1856. Seine Familie stammt von Wohlen im Kanton Bern. Der Vater war ein angesehener Jurist, Regierungsrat und Ständerat. Sahli war mit Bern stark verwurzelt. Berufungen nach auswärts lehnte er ab. Er starb am 28. April 1933.

Nach der Maturität, 1874, wurde er Mediziner. "Zur Medizin kam ich eigentlich zufällig », sagt er in seiner Selbstdarstellung ${ }^{1}$. Er hätte den Eindruck gehabt, daß er sich eigentlich in jedes beliebige Wissensgebiet durch intensive Beschäftigung damit hätte einleben können. Das wird schon so sein. Aber wahrscheinlich waren für die Berufswahl doch wichtige äußere Einwirkungen mit im Spiel: Einmal der damalige große Aufschwung der Naturwissenschaften. Durch die Virchowsche Zellularpathologie erfolgte die Loslösung von humoralen Anschauungen. L. Pasteur, der Chemiker, hatte 1857 die Gärung als fermentativ, durch lebende Organismen bedingt, erkannt ${ }^{2}$, im Gegensatz zu den chemischen Theorien von Liebig, und 1868 lebende Erreger auch als die Ursache der Seidenraupenkrankheit festgestellt. Die Entdeckungen von LISTER $1872^{3}$ waren gleichbedeutend mit dem Beginn der Antisepsis und Asepsis. Andererseits muß aber auch darauf hingewiesen werden, daß die damalige Berner Medizinische Fakultät sicherlich große Anziehungskraft besaß, mit THEodor Kocher ${ }^{4}$, der offenbar 1871 zum erstenmal über Kropfoperationen berichtete, Th. LANGHaNs ${ }^{5}$, der eben von Marburg nach Bern gekommen war, und QuincKe ${ }^{6}$, dem glänzenden Kliniker und Erfinder der Lumbalpunktion, dessen Spuren ich noch in Kiel angetroffen habe.

Sahli wurde also Mediziner und absolvierte sein Staatsexamen 1878. Die Dissertation ${ }^{7}$ bezog sich auf Die topographische Perkussion im Kindesalter, vor allem die Grenzperkussion, im Gegensatz zu der vorher üblichen vergleichenden Perkussion rechts und links. Es mußte sehr leise perkussiert werden. Sahli hat diese Technik auch beim Erwachsenen angewandt, und man erkennt jeden SahliSchüler an dieser Perkussionsweise.

${ }^{1}$ SAHLI, Die Medizin der Gegenwart in Selbstdarstellungen, herausgegeben von Grote, Leipzig 1925.

${ }^{2}$ Charles DhÉré, Louis Pasteur, son æuvre, sa psychologie, Fribourg 1923.

3 Trendelendurg. Joseph Listers erste Veröffentlichung über antiseptische Wundbehandlung, 1867, Barth, Leipzig 1912.

${ }^{4}$ Bonjour, Theodor Kocher, Bern 1950.

${ }^{5}$ C. Wegelin, «Prof. Th. Langhans», in Corr.-Bl. Schweizer Ärzte 1915, 52.

${ }^{6}$ Quincke, Die Technik der Lumbalpunktion, Berlin 1902.

${ }^{7}$ SAHLI, Die topographische Perkussion im Kindesalter, Bern 1882. 
Nach einem Jahr Kinderklinik unter Professor Demme kam Sahli an die Medizinische Klinik Bern unter Lichтнеiм, diesem sehr gestrengen Chef, der in seltener Weise höchste Feinheit des Geistes mit kraftvoller Entschiedenheit des Handelns in sich vereinte. Bern wurde aber nach kurzer Zeit mit Leipzig vertauscht. Lichtheim hatte offenbar erkannt, welche Kräfte in dem jungen Assistenten verborgen lagen, und ihn demjenigen Mann zugeführt, den er selbst als seinen Lehrer verehrte: Julius Cohnheim ${ }^{8}$, dem berühmten Experimentator und Pathologen. Unter dessen Leitung hatte Lichtheim in Breslau seine wichtigen Publikationen über das Ödem und den Blutkreislauf verfaßt, in Breslau waren die Arbeiten von Litten über Lungenembolie ${ }^{8}$ und dann vor allem diejenige von WELCH $^{9}$ über akutes Lungenödem entstand. In Leipzig wurde dann über ähnliche Themen weitergearbeitet. Mit seinem Chef war auch KARL WeIGERT ${ }^{10}$ nach Leipzig übergesiedelt. Er scheint Sahli besonders nahegestanden zu sein. Die drei Namen Lichtheim, Weigert, Cohnheim dürften die spätere Entwicklung von Sahli wesentlich mitbestimmt haben. An das Leipziger Wintersemester 1880/81 schloß sich eine Studienreise nach Wien sowie nach London, wo Sahli bei Gowers, Jackson, und nach Paris, wo er bei Charcot nach seinen Worten viel Anregung fand. Bei seiner Rückkehr nach Bern an die Lichtheimsche Klinik war die Basis für das Weitere geschaffen. Leipzig scheint wie ein Katalysator gewirkt zu haben. Wie auf einen Schlag erwachte in Sahli offenbar die Lust zu intensivstem wissenschaftlichem Arbeiten, packte ihn jenes feu sacré, das den Forscher in Atem zu halten pflegt. Rasch aufeinander erschienen in den folgenden Jahren Veröffentlichungen, die durch Originalität, Schärfe der Beweisführung, Gründlichkeit überraschten, diese eigenartige Sahlische Diktion, mit ihrer Klarheit der Fragestellung, dem Reichtum an Ideen, aber auch der Angriffslust, die den Leser gelegentlich erfreut.

Zuerst kommt der Leipziger Einfluß zur Geltung in der Publikation über das Lungenödem (1883) ${ }^{11}$ und wahrscheinlich auch bei der Abfassung der vorwiegend histologischen oder histologisch-technischen Arbeiten über die Anwendung der Borax-Methylenblaufärbung (1888) ${ }^{12}$, zum Nachweis von Mikroorganismen und als Kontrastfärbung bei der Histologie des zentralen Nervensystems. Die Sahlische wichtige Arbeit über Lungenödem nimmt sehr kritisch Stellung gegenüber Welch und damit auch gegenüber Cohnheim selbst, unter dessen Leitung die

${ }^{8}$ Julius Cohnнeim, Nekrolog für C. Weigert, in Gesammelte Abhandlungen 1906, II, 729.

${ }^{9}$ J.Conneem, Vorlesungen über allgemeine Pathologie, 2. Auflage, Berlin 1882.

${ }^{10}$ Ludwig Lichtheim, Nekrolog für C. Weigert, in Dtsch. Z. Nervenheilkunde 1904, 27, 345.

11 Sahli, "Zur Pathologie und Therapie des Lungenödems», in Arch. exp. Pathologie u. Pharmakologie, 1883433.

${ }^{12}$ SAHLI, «Über die Anwendung des Boraxmethylenblaus für die Untersuchung des Zentralnervensystems und für den Nachweis von Mikroorganismen", in Z. wiss. Mikroskopie 1885. 
experimentellen Untersuchungen von Sahli in Leipzig begonnen hatten. Das Lungenödem entspricht nicht immer einem Stauungshydrops, ein isoliertes Versagen des linken Ventrikels kommt nicht vor, weder im Tierversuch noch klinisch. Sahli hat immer klar Stellung bezogen. Er ging schon damals seinen eigenen Weg. Diese Unabhängigkeit, geistige Freiheit gab seinen Deduktionen auch die große Durchschlagskraft.

In der pathologisch-anatomischen Arbeit über die Lokalisation der kleinen Handmuskelkerne im Zentralnervensystem ${ }^{13}$ kommt er auch zu Schlüssen, welche mit der Auffassung des bekannten Prevost (1874) keineswegs übereinstimmten. Es fand sich trotz ganz isolierter degenerativer Atrophie des Daumens und Kleinfingerballens ein überraschend ausgiebiger Befund im Zervikalmark, welcher mit der Kompliziertheit der Handfunktion in Zusammenhang zu stehen schien.

Weitere Publikationen können nicht im einzelnen besprochen werden. Die Untersuchungen über Ankylostomumanämie ${ }^{14}$ bei Arbeitern des Gotthardtunnels, mit Beobachtungen über den Entwicklungsgang des Ankylostomum, Züchtungsversuchen, hatten weit abgeführt von früheren Problemen. Die Genauigkeit und Beharrlichkeit bei der Abfassung dieser Arbeit charakterisieren den Autor. An ein und derselben Sitzung des ärztlich-pharmazeutischen Bezirksvereins behandelte Sahli dann 1885 drei Themen, die jedes für sich später allgemeines Interesse fanden; diastolische akzidentelle Herzgeräusche ${ }^{15}$, Ektasie der Hautgefäße beiderseits im Bereich der untern Lungengrenze ${ }^{16}$, der Einfluß tabischer nervöser Krisen auf die Magensekretion ${ }^{17}$. Die ersten Publikationen über das Hämoglobinometer ${ }^{18}$, damals noch mit dem Gowerschen Instrument, mit Pikrinsäurekarmin als Testlösung, fällt in das Jahr 1887. Sahli weist auf sogenannte Pseudo-Anämien hin, bei speziellem Verhalten der Haut und Anomalien des peripheren Gefäßtonus. Auch mit chemischen Fragen befaßte sich Sahli da-

${ }^{13}$ SAHLI, "Zur Lehre von den spinalen Lokalisationen», Sektionsbefund bei einer alten Kinderlähmung mit eng lokalisierter Atrophie, in Dtsch. Arch. klin. Med. 1882, 1, 360.

${ }^{14}$ SAHLI, «Beitrag zur klinischen Geschichte der Anaemie der Gotthardtunnelarbeiter», in Dtsch. Arch. klin. Med. 1883, 32, 423.

${ }^{15}$ SAHLI, «Über das Vorkommen und die Erklärung acczidenteller diastolischer Herzgeräusche», in Corr.-Bl. Schweizer Ärzte 1885, 15, 257.

${ }^{16}$ SAHLI, «Über das Vorkommen und die diagnostische Bedeutung einer Zone ektasierter feinster Hautgefäße in der Nähe der untern Lungengrenze», in Corr.-Bl. Schweizer Ärzte 1885, 15, 135.

${ }^{17}$ SAHLI, «Über das Vorkommen abnormer Mengen freier Salzsäure im Erbrochenen bei den gastrischen Krisen eines Tabetikers mit Rücksicht auf die Frage nach Nerveneinflüssen auf die Sekretion des Magensafts», in Corr.-Bl. Schweizer Ärzte 1885, 15, 105.

${ }^{18}$ SAHLI, «Zur Diagnose und Therapie anämischer Zustände», in Corr.-Bl. Schweizer Ärzte 1886, 16, 554. 
mals eingehend. Die oft schlechte Verträglichkeit von salizylsaurem Natrium führte ihn, übrigens nach Konsultation von NENGKI, zu der Verwendung sogenannter «Salole» ${ }^{19}$, esterartiger Verbindungen von Phenolen und organischen Säuren. Derartige Komplexe sind nicht wasserlöslich, passieren den Magen unverändert, um erst vom Pankreassaft ähnlich den Fetten aufgespalten zu werden. Sahli behandelte infektiöse Darmaffektionen mit solchen Stoffen und sprach von einem unlöslichen Antiseptikum. Am Schluß dieser Schaffensperiode steht die 77 Seiten umfassende Monographie Über die modernen Gesichtspunkte in der Pathologie der Infektionskrankheiten ${ }^{20}$. Das Immunitätsproblem beschäftigte ihn in hohem Maße, Erinnerungen an Weigert und dessen Theorien über Entzündung lebten vielleicht in ihm auf, die Pasteurschen Erfolge mit abgeschwächten Vakzinen schienen neue therapeutische und prophylaktische Möglichkeiten zu geben, entsprechend der Kuhpockenschutzimpfung von Jenner (1796). Über Tuberkulose äußert sich Sahli in dieser Arbeit nicht. Der Tuberkelbazillus war zwar entdeckt, die künstliche Übertragung der Tuberkulose mit virulentem bazillärem Material bekannt (VILlemin 1865), die Arbeiten von R. Koch ${ }^{21}$ über Tuberkulin datieren aber erst vom Jahr 1890.

Die wissenschaftlichen Leistungen von Sahli hatten in Bern ihren Eindruck nicht verfehlt. Ludwig Lichtheim wurde 1888 nach Königsberg berufen. Hermann Sahli wurde zu seinem Nachfolger ernannt, im Alter von 32 Jahren.

Sahli verblieben von der Wahl zum Ordinarius 1888 bis zu seinem Tode 45 Jahre. Bewundernd sehen wir, wie er diese Zeit nutzte.

Sahli verfügte jetzt über eine Klinik, mit genügendem Krankenbestand, zuverlässigen Assistenten und einem Laboratorium, das er sich nach Bedürfnis schaffen konnte. Er war in jeder Hinsicht selbständig. Finanziell war er nicht besonders interessiert. Seine ganze Kraft konzentrierte sich auf den Unterricht und die Forschung auf dem ihm anvertrauten Gebiet der innern Medizin. «Mein Leben war ganz von der Medizin erfüllt», sagte er $1925^{1}$.

Man muß schon mit kräftigen Strichen zeichnen, um der Art seines Schaffens gerecht zu werden.

Eine ganz grundsätzliche Äußerung war schon die Publikation mit M. NENGKI, dem Vertreter der physiologischen Chemie in Bern, über « Enzyme in der Therapie»

${ }^{19}$ SAHLI, «Über die therapeutische Anwendung des Salols (des salizylsauren Phenols)», in Corr.-Bl. Schweizer Ärzte 1886, 16, 321.

${ }^{20}$ SAHLI, «Über die modernen Gesichtspunkte in der Pathologie der Infektionskrankheiten », in Volkmanns Sammlung klin. Vorträge, XI. Serie, 1888, 319/20, 284.

${ }^{21}$ R. Rochaldi, Robert Koch, der Schöpfer der modernen Bakteriologie, Stuttgart 1954. 
$(1890)^{22}$. Zelluläre Fermente werden neben der Phagozytose als eine der Waffen bezeichnet, deren sich der Tierkörper zur Bekämpfung von Infektionen bedienen könnte. Sahli hat 30 Jahre später die Antikörperbildung mit einer zellulären Sekretion verglichen, in offenbarer Fortentwicklung der in jungen Jahren schon konzipierten Idee.

Nach einem Vortrag über Auswaschung des menschlichen Organismus ${ }^{23}$ durch sukutane Zufuhr von physiologischer Kochsalzlösung, einer Weiterführung des Kroneckerschen Verfahrens ${ }^{24}$ mit intravenösen Infusionen, sprach Sahli am Schweizerischen ärztlichen Zentralverein 1891 über «Hirnchirurgische Operationen vom Standpunkt der inneren Medizin », ${ }^{25}$ mit Professor K RönleIN, Zürich, als chirurgischem Korreferenten. Es handelt sich im wesentlichen um ein Referat der vorliegenden Literatur mit Beifügung von Ausführungen über Hirnphysiologie und Hirnanatomie. Die einer Lokaldiagnose entgegenstehenden Schwierigkeiten werden erörtert. Man spreche besser von Foci (Brennpunkten) als von Zentren. Bei allgemeinem Hirndruck müsse auf eine sichere Lokaldiagnose verzichtet werden. Abszesse und auch die Jacksonsche Form der Epilepsie bieten operative Chancen. Zur Bekämpfung des Hirndrucks empfehle sich an Stelle der Trepanation eine Ventrikelpunktion. Die vorgeschlagene Ligatur der Karotis bei Hirnblutungen wird scharf abgelehnt. Das Referat richtet sich schließlich vor allem gegen Horsley und seine Thesen am Internationalen Kongreß in Berlin 1890. - 1892 behandelte Sahli vor dem Bernischen Bezirksverein das Thema des akuten Gelenkrheumatismus ${ }^{26}$. Trotz des Nachweises von Staphylokokken in den Geweben erwies sich die Gelenkflüssigkeit als steril. Die im Blut und in zahlreichen Organen in Reinkultur nachgewiesenen Erreger wurden von Sahli nicht als Saprophyten angesprochen, auch nicht als Ausdruck einer Sekundärinfektion. Der akute Gelenkrheumatismus erschien als Folge einer Staphylokokkeninvasion. Es sei das zwar nicht mit Sicherheit zu sagen, aber nicht unmöglich. Das Fehlen von Eiterungen in den Gelenken wurde mit der Annahme von abgeschwächten Eiterstaphylokokken zu erklären versucht. Man erkennt jetzt, von ferne betrachtet, gewisse Schwächen der Beweisführung. In seiner Selbstdarstellung ${ }^{1}$ (1925) geht Sahli aber noch weiter, der akute Gelenkrheumatismus wäre «nichts anderes als

${ }^{22}$ M. Nencki und H. SAHLI, «Die Enzyme in der Therapie», in Corr.-Bl. Schweizer Ärzte $1890,20,737$.

${ }^{23} \mathrm{~S}_{\text {AHLI, }}$ «Über Auswaschung des menschlichen Organismus und über den Wert und die Methoden der Wasserzufuhr in Krankheiten», in Corr.-Bl. Schweizer Ärzte 1890, 20, 545.

${ }^{24}$ KronecKer, «Kritisches und Experimentelles über lebensrettende Infusionen von Kochsalzlösung bei Hunden», in Corr.-Bl. Schweizer Ärzte 1886, 16, 447.

${ }^{25}$ SAHLI, «Über Hirnchirurgische Operationen vom Standpunkt der innern Medizin», in Volksmanns Sammlung klin. Vorträge 1891, 28, 275.

${ }^{26}$ SAHLI, «Zur Ätiologie des akuten Gelenkrheumatismus», in Corr.-Bl. Schweizer Ärzte $1892,22,22$. 
eine abgeschwächte Pyemie». In der ursprünglichen Publikation (1892) drückte er sich vorsichtiger aus...

Es folgte (1892) die interessante Demonstration eines Mannes mit Sternalspalte ${ }^{27}$. Bei geschlossener Glottis gab eine forcierte exspiratorische Bewegung des Thorax typisches Vesikuläratmen. Die damals übliche Auffassung des Vesikuläratmens als einem fortgeleiteten Trachealgeräusch (BAAs und Penzoldt) konnte nicht richtig sein.

Dann hielt Sahli seine großen Referate über «Perityphlitis und ihre medizinische und chirurgische Behandlung ${ }^{28}$ am 4. Schweizerischen Ärztetag 1892 in Genf und am Kongreß für innere Medizin in München (1893).

Der Begriff und das Vorkommen einer Stercoraltyphlitis werden sehr kritisch betrachtet und die übliche Behandlung mit hohen Einläufen und Abführmittel strikte abgelehnt. Die Appendizitis ist ein Analogon zur Angina und hat immer einen eitrigen Kern. Soweit befand sich Sahli ganz in Übereinstimmung mit der Chirurgie, abweichend war der Standpunkt dann aber in der Frage der Therapie. Von Sonnenberg stammte die These: «Die Pherityphlitiden gehören den Chirurgen. » Roux hatte in Genf erklärt: «La médication interne la plus stricte n'offre en face de ces cas suppurés, à aucun moment, une garantie certaine». Sahli hielt dem entgegen, nach eigener Statistik über 2713 Fälle in der Schweiz heilten $91 \%$ der nicht operierten Fälle. Es werde allerdings wohl zu wenig operiert. Wenn innert 3 Tagen nicht «rapide Besserung» eintritt, so solle man operieren. Sahli empfahl damals die Vornahme einer Probepunktion mit sehr dünner Nadel. Ich brauche nicht auszuführen, wie weit diese Auffassungen mit der gegenwärtigen Praxis in Widerspruch stehen. Sahli ist später an der Jahresversammlung der Schweizerischen Naturforschenden Gesellschaft in Bern $1914^{29}$ nochmals auf das Thema der Appendizitis zu sprechen gekommen. Er wandte sich jetzt mit noch vermehrter Schärfe gegen die Empfehlung einer Frühoperation. Es sei das Hyperchirurgie.

Nun kam die Herausgabe seines Lehrbuchs ${ }^{30}$. Sahli arbeitete wahrscheinlich schon lange an dessen Fertigstellung. 1894 erschien die erste Auflage. Es war das die große Tat, die Sahli weltberühmt machte. Der Verleger Deuticke, Wien, hatte

${ }^{27}$ SAHLI, «Über die Entstehung des Vesikuläratmens, eine neue Beobachtung über ein altes Thema», in Corr.-Bl. Schweizer Ärzte 1892, 22, 265.

${ }^{28}$ SAHLI, Die Perithyphlitis (Appendizitis) und ihre medizinische und chirurgische Behandlung. Schweizer Ärztetag, 28. Mai 1892, Genf, in Corr.-Bl. Schweizer Ärzte 1893, 19. - Über die Pathologie und Therapie der Perithyphlitis, Verhandlungen der deutschen Gesellschaft für innere Medizin, München, in Corr.-Bl. Schweizer Ärzte 1895, 18, 561. ${ }^{29}$ SAHLI, «Über den Einfluß der Naturwissenschaften auf die moderne Medizin», in Jahresversammlung der Schweizerischen Naturforschenden Gesellschaft, Bern 1914.

${ }^{30}$ SAHLI, Lehrbuch der klinischen Untersuchungsmethoden für Studierende und Ärzte, 1.Auflage, Deuticke, Wien 1894. 
ihn eingeladen, ihm ein Lehrbuch der klinischen Untersuchungsmethoden zu schreiben. Sali zögerte, entscheidend war schließlich «das eigene Bedürfnis». Es war ähnlich wie bei R. KocH, wo auch die Fertigstellung seiner «Untersuchung von pathogenen Organismen» zur festen Grundlage wurde für das ganze eigene Weiterarbeiten, nicht nur vom Außenstehenden als «Bibel der Bakteriologen» bezeichnet wurde (Bochalli) ${ }^{21}$. Sahlis Weitblick erkannte, wie sehr die umfassende Darstellung einer klinischen Untersuchungsmethodik die Führung seiner Klinik und den Unterricht erleichtern mußte, den Ärzten eine solide Basis für ihr eigenes praktisches Handeln geben mußte.

Eine Inhaltsangabe ist nicht möglich. Jedermann kennt die gewaltige Bedeutung dieses Werkes, das über die innere Medizin hinausragt, weil es nicht nur Methoden beschreibt, sondern gedanklich-theoretisch ganz umfassend das technische, das praktische Handeln mit der Theorie, d. h. dem Denken, verbindet. Das war eben auch der wesentliche Charakterzug des Verfassers selbst.

Die Schaffung des Lehrbuchs war gewissermaßen die befreiende Tat, die den Weg frei machte für ein konsequent vorwärtsgetriebenes konzentriertes, höchst produktives Arbeiten.

Man erkennt unschwer bestimmte Richtungen, verschiedene große Themen, gedankliche Komplexe, die immer wieder auftauchen, den Autor scheinbar dauernd in Atem halten. Es ist nicht die Vielheit der klinischen Kasuistik, die bestimmend war, vielmehr die Macht bestimmter Ideen, deren Richtigkeit empirisch zu beweisen war.

Sahli ging auf breiter Front vor. Fünf Gebiete der innern Medizin werden systematisch in Angriff genommen:

1. Die Diagnostik der Magenfunktion.

Prinzipiell wichtig war schon die Veröffentlichung über eine perorale Verwendung sogenannter Glutoidkapseln (1898) ${ }^{31}$, mit Formol gehärteter Gelatinekapseln, die vom Magensaft nicht angegriffen werden, aber unter Wirkung des Pankreassekrets relativ leicht zerfallen. Die pharmazeutische Industrie bedient sich dieses Prinzips noch jetzt. Sahli verdankte seine Entdeckung offenbar einem Chemiker der Firma Hausmann, St. Gallen, Dr. Weyland. Es dauerte Jahre mit zahllosen Versuchen, mit Hüllen aus verschiedenen Fett-, Wachs-, Harzsorten, mit auf verschiedener Weise gehärteter Gelatine, durch Chromsäure, Gerbstoffe, bis Sahli schließlich mit der Formolhärtung den gewünschten Erfolg hatte. 1905 folgte dann die Publikation des Desmoidverfahrens ${ }^{32}$ zur Prüfung der Magenfunktion. Es wurden nach langwierigen Vorversuchen Beutelchen von Gold-

${ }^{31}$ SAHLI, «Diagnostische und therapeutische Anwendung der Glutoidkapseln», in Corr.-Bl. Schweizer Ärzte 1898, 10, 289; Dtsch. Med. Wschr. 1897, 1.

32 SAHLI, «Über Prüfung des Magenchemismus unter natürlichen Verhältnissen und ohne Anwendung der Schlundsonde. Die Desmoidreaktion, eine neue Untersuchungsmethode», in Corr.-Bl. Schweizer Ärzte 1905, 8, 241. 
schlägerhaut verwendet, mit Jodoform gefüllt und mit Rohkatgut zugebunden. Nur dieses ungekochte Bindegewebe erfüllte die Forderung, von Trypsin nicht, von Pepsin aber gelöst zu werden. Die freigewordene jodhaltige Substanz wurde im Speichel nachweisbar. Gegenwärtig gibt das Röntgenverfahren, die fraktionierte Untersuchung des Magensafts mit der EinHoRNschen Schlundsonde wohl verläßlichere Resultate. Die erwähnten Publikationen sind aber charakteristisch für die Arbeitsweise von Sahli. Er verfügte über eine erstaunliche Geduld und Beharrlichkeit.

Später wandte er sich ebenfalls dem Schlundsondenverfahren zu, um Sekretion und Motorik des Magens quantitativ beurteilen zu können, mit Verwendung des sogenannten Suppenprobefrühstücks. Noch im Jahre 1924 publizierte er ein Verfahren zur Bestimmung der Wasserstoffionenkonzentration des Magensafts ${ }^{33}$ durch Verwendung von Methylviolett als einzigem Indikator.

2. Die Reihe der Arbeiten hämatalogischer Art beginnt mit der Demonstration eines abgeänderten Gowerschen Hämoglobinometers ${ }^{34}$ am Kongreß für innere Medizin 1902, mit salzsaurem Hämatin als Testlösung. Dann folgte die Monographie über Hämophilie ${ }^{35}$ (1905), mit weiteren Beiträgen zur Lehre von der Hämophilie $1910^{36}$.

Schon 1894 hatte Sahli eine Dissertation von EgUET ${ }^{37}$ vorgelegt, welche neben mechanischen Faktoren die Bedeutung chemischer Vorgänge bei der Gerinnung bewies. Die Hämophilie wurde damals einfach zu den hämorrhagischen Diathesen gerechnet. Sahli zeigte dem gegenüber, in Bestätigung älterer Angaben von Grandidier (1877), Lossen (1876), Wright (1893), daß das Wesentliche eine streng spezifische Störung des Gerinnungschemismus sei. Erythrozyten, Leukozyten, Fibringehalt des Blutes, Plättchenzahl, Wassergehalt, Alkalität, Trockenrückstand, Gefrierpunktserniedrigung waren normal, einzig die Gerinnungsfähigkeit des Bluts erwies sich als extrem niedrig. Sehr auffallend war die Beobachtung, daß das aus einer Verletzung schon während längerer Zeit tropfende Blut normale oder sogar erhöhte Gerinnungsfähigkeit besaß. Der Organismus reagierte offenbar auf die Blutung mit einer Gegenaktion. Es mußte ferner neben der humoralen Herabsetzung der Gerinnungsfähigkeit auch noch eine abnorme

${ }^{33}$ SAHLI, «Über die Bestimmung der freien Säure des Magensaftes (der H-Ionenkonzentration) durch Titration der Indikatorlösung unter Verwendung von Methylviolett und Lackmus», in Schweiz. Med. Wschr. 1924, 1.

${ }^{34}$ SAHLI, «Über ein einfaches und exaktes Verfahren der klinischen Hämometrie», in Verh. dtsch. Ges. innere Med. 1902, 20, 230.

${ }^{35}$ SAHLI, «Über das Wesen der Hämophilie», in Z. klin. Med. 1905, 56.

${ }^{36}$ SAHLI, «Weitere Beiträge zur Lehre von der Hämophilie», in Dtsch. Arch. klin. Med. 1910, 99.

${ }^{37}$ Eguet, «Über den Einfluß des Blutegelinfuses auf die Thrombenbildung», in Mitt. Kliniken u. Instituten der Schweiz, II. Reihe, 1894, 4. 
Qualität der Gefäßwandung vorhanden gewesen sein. Durch spezielle Versuchsanordnungen glückte Sahli der Beweis, daß es sich nicht um eine schlechtere Kontraktilität der Gefäße handelte, sondern um einen Mangel an Thrombokinase: «Es wäre nicht undenkbar», sagt Sahli, «daß der hamophile Organismus sich dadurch von der Norm unterscheidet, daß infolge einer vererbbaren Fehlerhaftigkeit des Keimplasmas seine Zellen ganz allgemein weniger leicht Thrombokinase abgeben oder davon weniger enthalten.» - Die 53 Seiten umfassende Arbeit ist ein hervorragendes Beispiel für die Wirksamkeit einer sorgfältig durchgeführten rein klinischen Analyse.

3. Eine Hauptleistung ist dann der in verschiedenen Ansätzen immer wieder aufgenommene Kampf gegen die Tuberkulose, namentlich die Ausarbeitung einer rationellen Tuberkulintherapie.

Die verheerende Wirkung der sogenannten I. Tuberkulinära ist bekannt. Die von Robert Koch am Internationalen medizinischen Kongreß in Berlin (4. August 1890) vorsichtig gehaltenen Äußerungen ${ }^{21}$ und seine bald darauf folgenden Mitteilungen in der Deutschen Medizinischen Wochenschrift (1890) «über ein Heilmittel gegen Tuberkulose» waren mißverstanden worden. Man dosierte zu hoch. R.Koch hatte zum Zweck einer künstlichen Immunisierung des an Tuberkulose Erkrankten Glycerinextrakte aus Reinkulturen von Tuberkelbazillen subkutan injiziert.

1906 erschien die Monographie von Sahli « Über das Wesen des Tuberkulins und der Tuberkulinwirkung, sowie Tuberkuloseheilung und Tuberkuloseimmunität» ${ }^{38}$. Sahli widmete das Buch seinem Lehrer Lichtheim. Es hat vier Auflagen erlebt und ist zu einer Darstellung der ganzen Lehre von der Tuberkuloseimmunität geworden.

Ich kann nur einige wesentliche Punkte festhalten: Tuberkuline sind Proteinkörper, die den Organismus zur Abgabe albuminolytischer (nicht bakteriolytischer) Lysine anregen. Die entstehenden Abbauprodukte sind - wenigstens zum Teil - toxisch. Außerdem entstehen wahre Antitoxine. Es gibt eine Schutzwirkung auf dem Weg über eine Überempfindlichkeit der Gewebe. Wenn die Tuberkulintherapie auf kleinste Dosierungen und kaum wahrnehmbare Reaktionen sich einstelle, so käme eine solche Therapie dem Geschehen bei der Naturheilung der Tuberkulose nahe. Sahli erwähnt, daß schon Lichtheim die Möglichkeit einer reaktionslosen Tuberkulinbehandlung erkannt hätte.

Sahli behandelt auch ausführlich die Frage einer prophylaktischen Immunisierung mit wenig virulenten lebenden Bazillen. «Man sollte aber glauben, daß sich Immunität auch durch die Tuberkulinwirkung allein erzielen lasse. Mit Tuberkulin lassen sich experimentell typische Tuberkel erzeugen.»

${ }^{38}$ SAHLI, Über Tuberkulinbehandlung, 3. Auflage, «Über das Wesen des Tuberkulins und der Tuberkulinwirkung sowie der Tuberkuloseheilung und Tuberkuloseimmunität», Benno Schwabe, Basel 1910. 
Über die diagnostische Verwertung des Tuberkulins äußert sich Sahli nur kurz und im wesentlichen ablehnend. Die subkutane Einverleibung von Tuberkulin zu diagnostischen Zwecken wird völlig verworfen. Die Pirquetsche Kutanprüfung gibt leicht falsche Resultate.

1920 hielt Sahli seinen vielbeachteten Vortrag: «Über das Wesen und die Entstehung der Antikörper. ${ }^{39}$ Er galt hauptsächlich einer Kritik der EHrLICHschen Seitenkettentheorie. Das Protoplasma sei eine Kolloidstruktur, nicht vergleichbar mit einer chemischen Verbindung. Der Begriff der Regeneration sei ein morphologischer, chemische Seitenketten regenerierten sich nicht. Man könne sich die Enstehung von Antikörpern ähnlich vorstellen wie eine zelluläre Sekretion «bei eintretendem Bedürfnis». Die Zelle reagiere grundsätzlich auf bestimmte Blutzusammensetzungen. Präformiert im Blut vorhandene Antikörper könnten durch das Antigen gebunden werden, worauf der Körper mit Neubildung derselben reagiert.

Sahli ist 1924 in seinem Vortrag «über den Kampf des Organismus gegen den Tuberkulosebazillus ${ }^{40}$ an der Konferenz der internationalen Union gegen die Tuberkulose in Lausanne nochmals auf die Frage der Tuberkulintherapie zurückgekommen. Er empfahl jetzt das Subepidermale Verfahren. Nach der Weigertschen Entzündungstheorie diene die entzündliche Reaktion der Beseitigung entzündlicher Gewebszerfallsprodukte. Zelluläre zweckmäßige Reaktionen seien die Phagozytose durch Lymphozyten (Bergell) und Histiozyten (Aschoff), auf chemischem Wege wirke die Immunisierung. Das Beranecksche Tuberkulin enthalte Endo- wie Exotoxine des Tuberkelbazillus, wirklich die Summe der wichtigsten Tuberkuloseantigene, und sei deshalb für eine immunisierende Therapie besonders zu empfehlen.

Es ist nicht der Ort und der Moment, hier kritische Bemerkungen vorzubringen. Ohne jeden Zweifel hat sich Sahli durch seine Tuberkulinarbeiten um die Sache der Tuberkulosebekämpfung außerordentlich verdient gemacht. Wenn man jetzt in der Lage ist, den Bazillus selbst zu stabilisieren oder gar abzutöten, so kommt einer mit dieser antibazillären Therapie kombinierten, die natürlichen Abwehrkräfte des Körpers stimulierenden Tuberkulintherapie immer noch große Bedeutung zu.

4. Ein weiteres, Sahli in den letzten Dezennien seiner Tätigkeit stark beschäftigendes Thema ist das der Hämodynamik.

Sahli ist schon 1901 mit einem Referat über Herz- und Vasomotorenmittel ${ }^{41}$ am Kongreß für innere Medizin in Wiesbaden hervorgetreten. Er befaßte sich hier vor

39 SAHLI, «Über das Wesen und die Entstehung der Antikörper », in Schweiz. Med. Wschr. 1920, 50, 1129.

40 SAHLI, «Über den Kampf des Organismus gegen den Tuberkelbazillus », IV. Konferenz der Internationalen Union gegen die Tuberkulose, Lausanne, in Schweiz. Med. Wschr. $1924,32,713$.

41 SAHLI, «Herz- und Vasomotorenmittel», in Verh. dtsch. Ges. innere Med. 1901, 19, 46. 
allem mit der Digitalisfrage. Sahli rechnete noch mit einer «ansaugenden diastolischen» Wirkung der Droge. Digitalis wurde damals nur peroral verwendet, als Tinktur oder Infus. Allgemeines Interesse erweckte die Feststellung, daß der Blutdruck bei Hypertonikern unter Digitaliseinfluß zurückgehe; ein Teil der Hypertonie schien durch kardiale Insuffizienz, zerebrale Ischämie bedingt zu sein, wie bei der Erstickung. In der Diskussion wurde Digitoxin als das beste der Digitalispräparate bezeichnet. Sahli und auch NAUNYN warnten aber davor: Digitoxin sei außerordentlich heftig wirkend und hätte relativ starke gefäßkontrahierende Effekte. Eine völlig richtige Beurteilung der Sachlage.

Ich erwähne dann kurz die Arbeiten über Sphygmographie ${ }^{42}$ und arterielle Tonometrie $^{43}$. Sahli ersetzte die sonst übliche Riva-Rocci-Manchette durch eine peripher einwirkende Druckpelotte, entsprechend dem BAschschen Verfahren; die Werte für maximalen und minimalen Druck wurden mittels eines Quecksilbermanometers bestimmt. Dabei ergaben sich nun Schwierigkeiten bei der Ermittlung des Minimaldrucks. Sahli ging deshalb über zur Verwendung eines mit Alkohol gefüllten trägheitsfreien Indexmanometers ${ }^{44}$, ein wichtiger Entschluß, weil der Minimaldruck mit einem solchen «Manometer» wesentlich tiefer gefunden wird als mit einem $\mathrm{Hg}$-Manometer, was wieder theoretisch große Konsequenzen hatte. Sahli kam in Gegensatz zu der herrschenden Anschauung, hielt aber noch 1923 an seinen Auffassungen fest ${ }^{45}$. Das Sahlische «absolute Sphygmogramm ${ }^{46}$ ist eine schematische graphische Darstellung von Maximal-, Minimal(und Optimal-) Druck als Ordinate, und der Zeit einer Systolendauer als Abszisse.

Von großem Interesse ist die Sahlische Sphygmobolometrie. Schon $1907^{47}$ machte Sahli darauf aufmerksam, daß die Ermittlung des Blutdrucks allein zur Beurteilung der Zirkulationsverhältnisse nicht genüge; es müsse vor allem der Energiewert des Einzelpulses bekannt sein, das Produkt Volum (Füllung) mal Druck, entsprechend dem Produkt Stromstärke mal Spannung. Die technische Ausgestaltung und auch die theoretische Begründung des Verfahrens haben verschiedene Phasen durchgemacht, erst 1920 konnte das «definitive» Instrumen-

${ }^{42}$ SAHLI, Lehrbuch der klinischen Untersuchungsmethoden für Studierende und Ärzte, 6. Auflage, 1913, 120-83.

${ }^{43}$ SAHLI, Lehrbuch der klinischen Untersuchungsmethoden für Studierende und Ärzte, 6. Auflage, 1913, 183-212.

${ }^{44}$ SAHLI, «Indexmanometer», in Lehrbuch der klinischen Untersuchungsmethoden für Studierende und Arzte, 6. Auflage, 1913, I, 221.

${ }^{45}$ SAHLI, «Minimaler Blutdruck», in Lehrbuch der klinischen Untersuchungsmethoden für Studierende und Ärzte, 6. Auflage, 1920, II, 1278; Wiener Arch. innere Med. 1922, 4, und 1923,6 .

${ }^{46}$ SAHLI, «Über das absolute Sphygmogramm (Drucksphygmogramm)», in Dtsch. Arch. klin. Med. 1904, 81.

${ }^{47}$ SAHLI, «Sphygmobolometrie», in Dtsch. Med. Wschr. 1907, 16. 
tarium herausgegeben werden ${ }^{48,49}$. Es wird dabei das systolische Pulsvolum mittels der mit Alkohol gefüllten Indexkapillare direkt in Kubikzentimeter gemessen und der Energiewert durch Multiplikation mit dem jeweiligen Pelottendruck errechnet.

Das Verfahren begegnete viel Kritik, weniger aus technischen Gründen als durch den Hinweis darauf, daß die erhaltenen Pulswerte aus rein peripheren Gründen stark wechseln könnten. Sahli konstruierte das Arteriometer ${ }^{50}$, welches das innere Kaliber der Arterie bis auf $1 / 10 \mathrm{~mm}$ genau zu messen vermochte, um damit das gefundene Pulsvolum auf Einheitswerte zu reduzieren. Es gibt nun aber in praxi keine ganz sichern Normalwerte. Der Haupteinwand war aber der, der Puls sei weniger Füllung (Bolos) als Wellenbewegung. Die Pulsgröße stehe mit der Größe des Herzschlagvolums in keiner festen Beziehung. Das von Отто Frank, München, inaugurierte und durch Wezler, Brömser ausgebaute Verfahren der «Windkessel»-Analyse ${ }^{51}$ bekam praktisch schließlich doch das Übergewicht: Die Bestimmung von Pulswellengeschwindigkeit und Eigenschwingung des arteriellen Systems läßt die Größe des aortalen Elastizitätkoeffizienten, und die Kenntnis dieses Faktors zusammen mit der Druckamplitude das systolische Schlagvolum zurzeit mit befriedigender Genauigkeit errechnen.

Die von Sahli vorgenommene U̇bertragung physiologischer Erkenntnisse, einer dynamischen energetischen Betrachtungsweise der Blutzirkulation, auf die Klinik war neu und hat außerordentlich anregend gewirkt.

5. Ich komme nun zum letzten Kapitel, den Leistungen von Sahli als Neurologe. Er vertrat immer die Ansicht, die Neurologie gehöre zur innern Medizin, man vergleiche z. B. die Diskussion mit Oтto Veraguth am Ärztetag in Bern 1952.

Als junger Assistent hatte er sich mit pathologisch-anatomischen Untersuchungen des zentralen Nervensystems ${ }^{12}$ befaßt. Seinen Vortrag über hirnchirurgische Operationen ${ }^{25}$ habe ich erwähnt. In der Festschrift für Lichtheim steht der Beitrag über kortikale Lokalisationen der konjugierten Augenbewegungen $^{53}$ (1905). Das Lehrbuch stellt die Untersuchung des Nervensystems auf mehr

${ }^{48}$ SAHLI, «Über die jetzige Form der Volumbolometrie », in Schweiz. Med. Wschr. 1920, 1, 2.

${ }^{49}$ SAHLI, "Sphygmobolometrie, das Volumbolometer», in Lehrbuch der klinischen Untersuchungsmethoden für Studierende und Ärzte, 6. Auflage 1920, II, 1298.

${ }^{50}$ SAHLI, «Über die objektive sphygmographische Messung des Arterienlumens (sphygmographische Arteriometrie) als Hilfsmittel und Schlußstein der dynamischen Pulsuntersuchung », in Schweiz. Med. Wschr. 1922, 6.

${ }^{51}$ WeZLER und BöGER, «Die Dynamik des arteriellen Systems», in Ergebnisse Physiologie 1939, 41, 292.

52 SAHLI, «Über den neurologischen Unterricht an den Schweizer Universitäten», in Corr.-Bl. Schweizer Ärzte 1912, 6, 195.

${ }^{53}$ SAHLI, «Beitrag zur kortikalen Lokalisation der konjugierten Seitwärtsbewegung der Augen und des Kopfes», in Dtsch. Arch. klin. Med.1905, 86. (Festschrift für Lichtheim.) 
als 600 Seiten dar. Gewissermaßen abschließend trat er nun nach langer Pause noch mit zwei großen Leistungen hervor, dem Vortrag über Neurosen (1922) ${ }^{54}$ und demjenigen über die Beziehungen des Geistes zum Körper (1931) ${ }^{55}$.

In der ersten Arbeit präzisiert er seine Ansichten speziell im Hinblick auf die Hysterie. Sie ist seiner Ansicht nach anatomisch lokalisiert. Sie entspricht einem abnormen Geschehen im Bereich des kortikalen Assoziationssystems von Flechsig, jenes Gebiets, wo sich «die geistigen und körperlichen Funktionen gewissermaßen die Hand reichen ». Die Aufgabe dieses «Interzentralsystems» ist, abgesehen von den geistigen Vorgängen des Denkens, Fühlens, Wollens und der Wahrnehmung, der anatomische Anschluß von Sensibilität und Motilität, der Projektionssysteme, an den Bewußtseinsinhalt. Hierfür sei das Interzentralsystem gewissermaßen der Rangierbahnhof. Die Hysterie erscheint als eine funktionelle Herdläsion. Die hysterischen Symptome sind im wesentlichen körperliche Äußerungen mit oder ohne psychische Parallel-Prozesse. Sahli nennt die Hysterie «somatische Interzentralneurose».

Man hätte jetzt wahrscheinlich noch den Einfluß dienzephaler und thalamischer Strukturen mitzuberücksichtigen.

Der zweite Vortrag bezieht sich auf das alte Thema «Seele und Körper». Es ist das die letzte Arbeit von Hermann Sahli, zwei Jahre vor seinem Tode geschrieben. Sie erscheint wie eine Art Vermächtnis.

Die Leistungen des geistigen und des körperlichen sind inkommensurabel: Alles Körperliche spielt sich in Raum und Zeit ab, das Geistige, d. h. Vorstellungen, Begriffe besitzen keinen Raum-Zeit-Charakter. Die zwischen beiden unleugbar bestehenden kausalen Beziehungen werden aber bei einer energetischen Auffassung der geistigen Leistungen verständlich.

Das Geistige ist als eine «spezielle Energieform » zu betrachten. Das kalorische Äquivalent der geistigen Energie ist klein, ihr Ausnutzungskoeffizient aber groß. Der geistige Impuls erfährt auf seinem Weg zur Peripherie eine Energietransformation. Die geistige Energie eines Willensimpulses bedingt das explosive Freiwerden von physikalischer Energie im Muskel durch Auslösung, gewissermaßen durch «Zündung». Die geistige Energie wird so zur Ursache für das Freiwerden von materieller Energie.

Ich habe alle diese Sätze wörtlich zitiert. Es ist erstaunlich, wie sehr die Richtigkeit der Sahlischen Thesen durch die moderne Physiologie bestätigt wird. Man kann wohl sagen, daß Sahli intuitiv in glänzender Weise existente Mechanismen vorausgeahnt hat.

${ }^{54}$ SAHLI, «Über den Begriff und das Wesen der sogenannten allgemeinen Neurosen», in Verhandlungen der Schweizerischen Naturforschenden Gesellschaft 1922, 25. August 1862.

${ }^{55}$ SAHLI, «Über die Beziehung des Geistes zum Körper. Fällt das Geistige in den Bereich der Energetik? », in Schweiz. Med. Wschr. 1931, 3, 49. 
Ein genialer Organismus läßt gewisse immanente, gewissermaßen zwingende Richtlinien seines Denkens und Handelns erkennen. Louis Pasteur, Robert Koch sind Beispiele dafür. Große Leistungen sind ohne konsequenten Aufbau nicht möglich oder wenigstens die Ausnahme.

Überblicken wir das Lebenswerk von Hermann Sahli, so scheinen uns drei Männer stark bestimmend gewesen zu sein: L. Lichtheim in Bern, C. Weigert und J. Cohnheim in Leipzig. Dem faszinierenden Einfluß von Lichtheim konnte man sich nicht entziehen. Während der Assistentenzeit von Sahli publizierte Lichtheim seine berühmte Abhandlung über Aphasie; sie wird auf seine Assistenten großen Eindruck gemacht, «Engramme» hinterlassen haben. Daher sicherlich seine Vorliebe für die Neurologie. C. Weigert stand zwischen der Naturphilosophie früherer Zeiten und exakter Naturwissenschaft. Er war der hervorragende Histologe, aber auch ein Denker überlegener Art. «Er suchte überall die Wurzeln, aus denen die Dinge emporsprießen, in jeder Einzelerscheinung das ihr innewohnende Gesetz, das Allgemeine», sagt Rieder ${ }^{56}$ in der Einleitung zu den gesammelten Werken von Weigert. Wer denkt da nicht an Hermann Sahli? Weigert befaßte sich in Leipzig vorwiegend mit dem Entzündungsproblem, der primär nekrotisierenden Wirkung bakterieller Gifte und der sekundären Ersatzwucherung, überhaupt den regeneratorischen Kräften der Gewebe. Sahli lebte auch in solchen Vorstellungen, und von Cohnheim hatte Sahli vielleicht seine Vorliebe für die Bearbeitung zirkulatorischer Fragen.

Nach Heisenberg ${ }^{57}$ ist abendländische Kultur: die Verbindung von prinzipieller Fragestellung und praktischem Handeln. Die Griechen hatten mehr als andere Völker die Fähigkeit, eine gestellte Frage ins Prinzipielle wenden zu können. Sahli bewunderte die antike Geistesbildung ${ }^{58}$. Er erscheint uns wie die Verkörperung alter Kultur. Er konnte prinzipiell denken, aber dann auch mit ungeheurer Kraftentfaltung handeln.

Sahli war der Lehrer der jungen Mediziner während 41 Jahren. Sein Bild hängt in manchem Laboratorium. Seine Vorlesungen waren klar, von tiefem Ernst getragen, äußerst wirkungsvoll. Seine große Gestalt steht mahnend vor uns: Er soll unser Vorbild bleiben.

Walter Frey, Bern/Oberhofen

${ }^{56}$ C. Weigert, Gesammelte Abhandlungen, unter Mitwirkung von Ludwig Edinger und Paul Ehrlich herausgegeben von Robert Rieder, Springer, Berlin 1906.

${ }^{57}$ Heisenberg, Das Naturbild der heutigen Physik, Rowohlt, Hamburg 1956.

${ }^{58}$ SAHLI, Einige Bemerkungen zum Wert des altsprachlichen Unterrichts im Lichte der Psychologie des Denkens, Benno Schwabe, Basel 1923. 